\title{
Supporting Information for "Extrapolative Bayesian Optimization with Gaussian Process and Neural Network Ensemble Surrogate Models"
}

Yee-Fun $\operatorname{Lim}^{1}$, Chee Koon $\mathrm{Ng}^{1}$, US Vaitesswar ${ }^{1}$, and Kedar Hippalgaonkar ${ }^{1}$

${ }^{1}$ Affiliation not available

July 20, 2021

\section{Supporting Information}

Yee-Fun Lim, ${ }^{*}$ Chee Koon Ng, US Vaitesswar, Kedar Hippalgaonkar*

\section{Contents}

Figures: S1 - S13

Table: S1

Figures with Data and Code:

1. Concrete dataset machine learning predictions using Neural Ensemble Regressor

2. Visualizing the extrapolative performance of the Neural Ensemble Regressor

3. Visualizing the concrete Bayesian Optimization in 1D using the Neural Ensemble as the surrogate model

Data:

1. Concrete compressive strength dataset

2. Thermoelectrics power factor dataset

3. Power plant output dataset

Code:

1. Concrete optimization (Jupyter ipython notebook)

2. Thermoelectrics optimization (Jupyter ipython notebook)

3. Power plant optimizaton (Jupyter ipython notebook)

4. Neural Ensemble regressor (python file)

5. Neural Dropout regressor (python file) 


\section{Figures}

\begin{tabular}{|c|c|c|c|c|c|c|c|c|c|}
\hline & 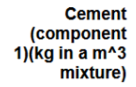 & 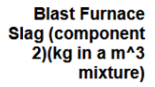 & $\begin{array}{r}\text { Fly Ash } \\
\text { (component } \\
3)(k g \text { in a m^3} \\
\text { mixture) }\end{array}$ & 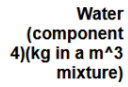 & $\begin{array}{r}\text { Superplasticizer } \\
\text { (component 5)(kg in } \\
\text { a } \mathrm{m}^{\wedge} 3 \text { mixture) }\end{array}$ & $\begin{array}{r}\text { Coarse } \\
\text { Aggregate } \\
\text { (component 6)(kg } \\
\text { in a m^3 mixture) }\end{array}$ & 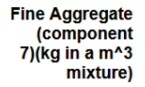 & $\begin{array}{c}\text { Age } \\
\text { (day) }\end{array}$ & $\begin{array}{r}\text { Concrete } \\
\text { compressive } \\
\text { strength(MPa, } \\
\text { megapascals) }\end{array}$ \\
\hline 0 & 540.0 & 0.0 & 0.0 & 162.0 & 2.5 & 1040.0 & 676.0 & 28 & 79.986111 \\
\hline 1 & 540.0 & 0.0 & 0.0 & 162.0 & 2.5 & 1055.0 & 676.0 & 28 & 61.887366 \\
\hline 2 & 332.5 & 142.5 & 0.0 & 228.0 & 0.0 & 932.0 & 594.0 & 270 & 40.269535 \\
\hline 3 & 332.5 & 142.5 & 0.0 & 228.0 & 0.0 & 932.0 & 594.0 & 365 & 41.052780 \\
\hline 4 & 198.6 & 132.4 & 0.0 & 192.0 & 0.0 & 978.4 & 825.5 & 360 & 44.296075 \\
\hline
\end{tabular}

Figure. S1. Snapshot of the concrete compressive strength dataset, showing the inputs for composition and processing parameters and the corresponding output target of the compressive strength. There are 8 input columns in total.

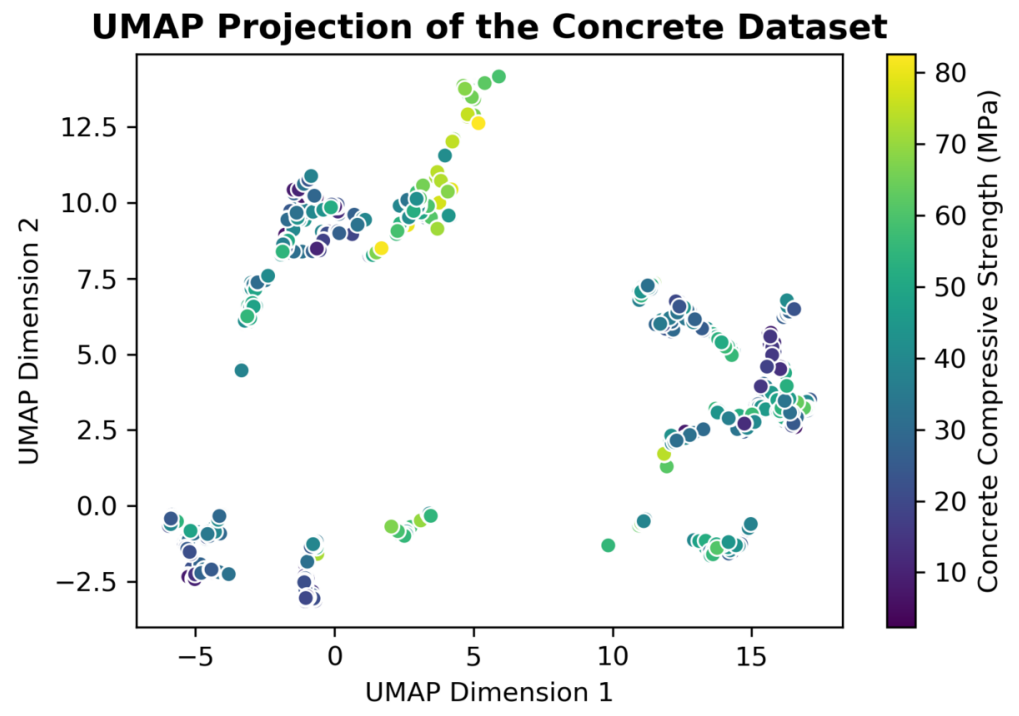

Figure. S2. UMAP projection of the concrete dataset, showing in reduced 2D space the data distribution and the spread in the concrete compressive strength values. 

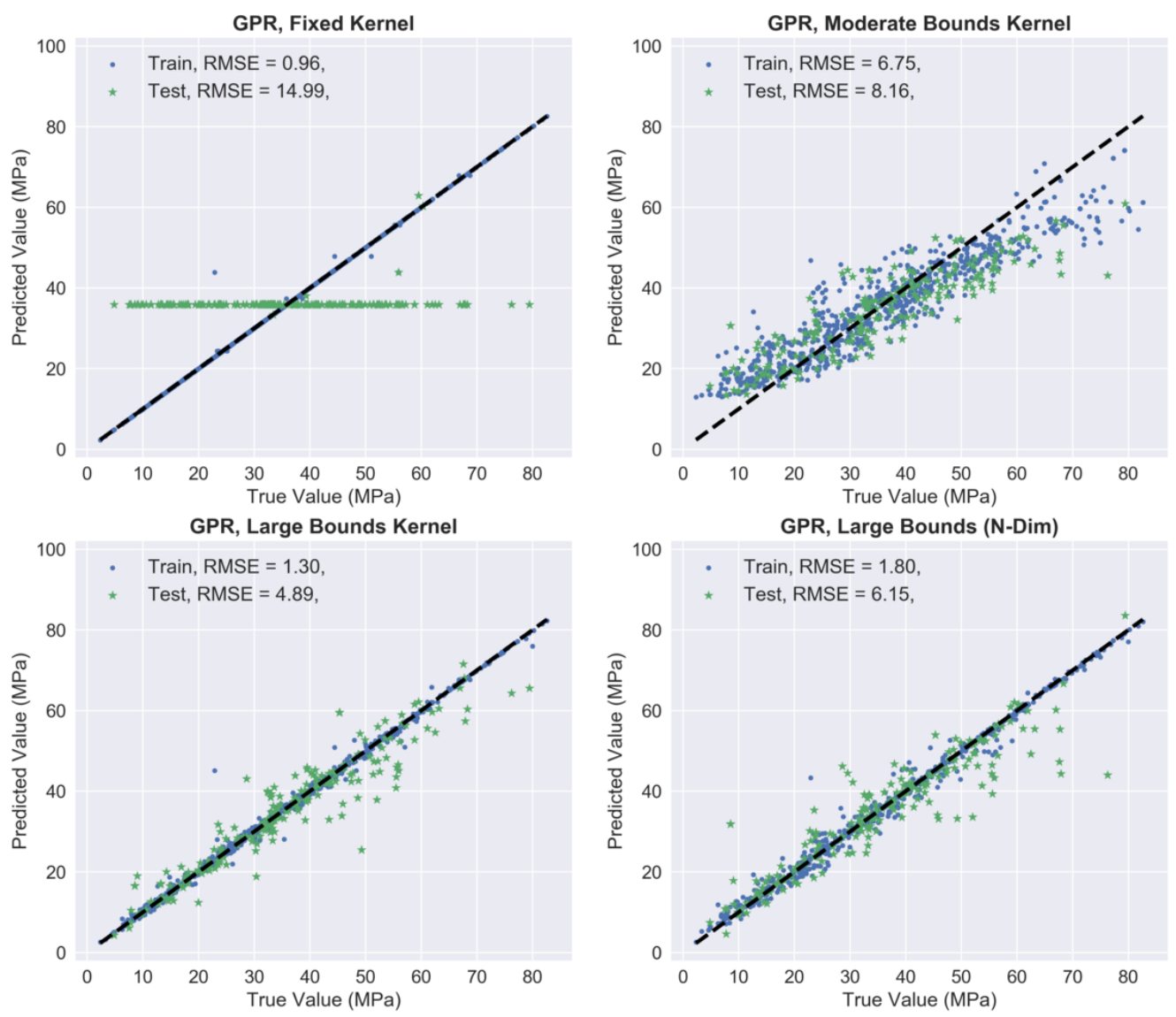

Figure. S3. Performance of the Gaussian Process Regressor on the concrete dataset with different choice of the range for the kernel bounds: fixed (top left), moderate (top right), large (bottom left), large and varying independently for each input dimension (bottom right). 


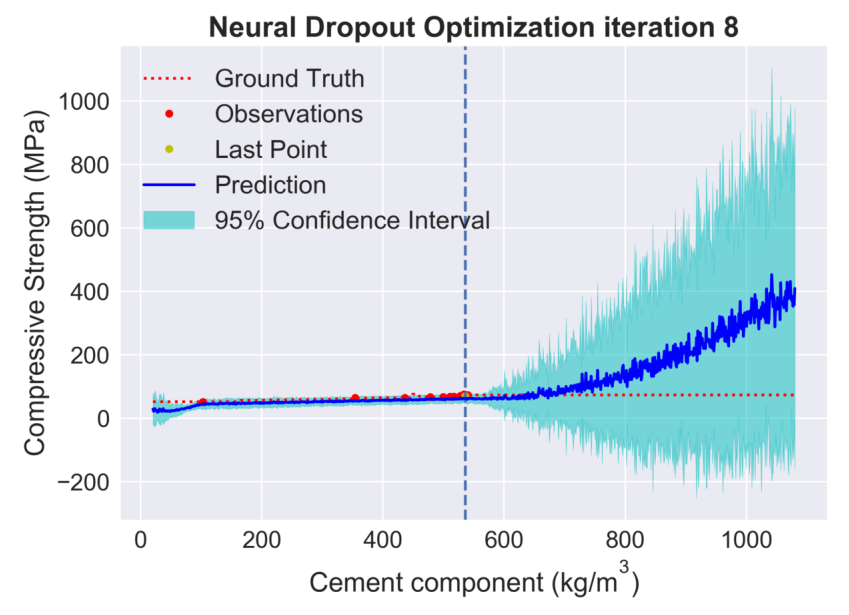

Figure. S4. Visualization of the prediction and uncertainty manifolds for the Neural Dropout surrogate model, with the range expanded beyond the bounds of the dataset. Note that within the dataset bounds (50-600), the uncertainty is relatively constant, but it starts to increase in the regions outside the bounds.
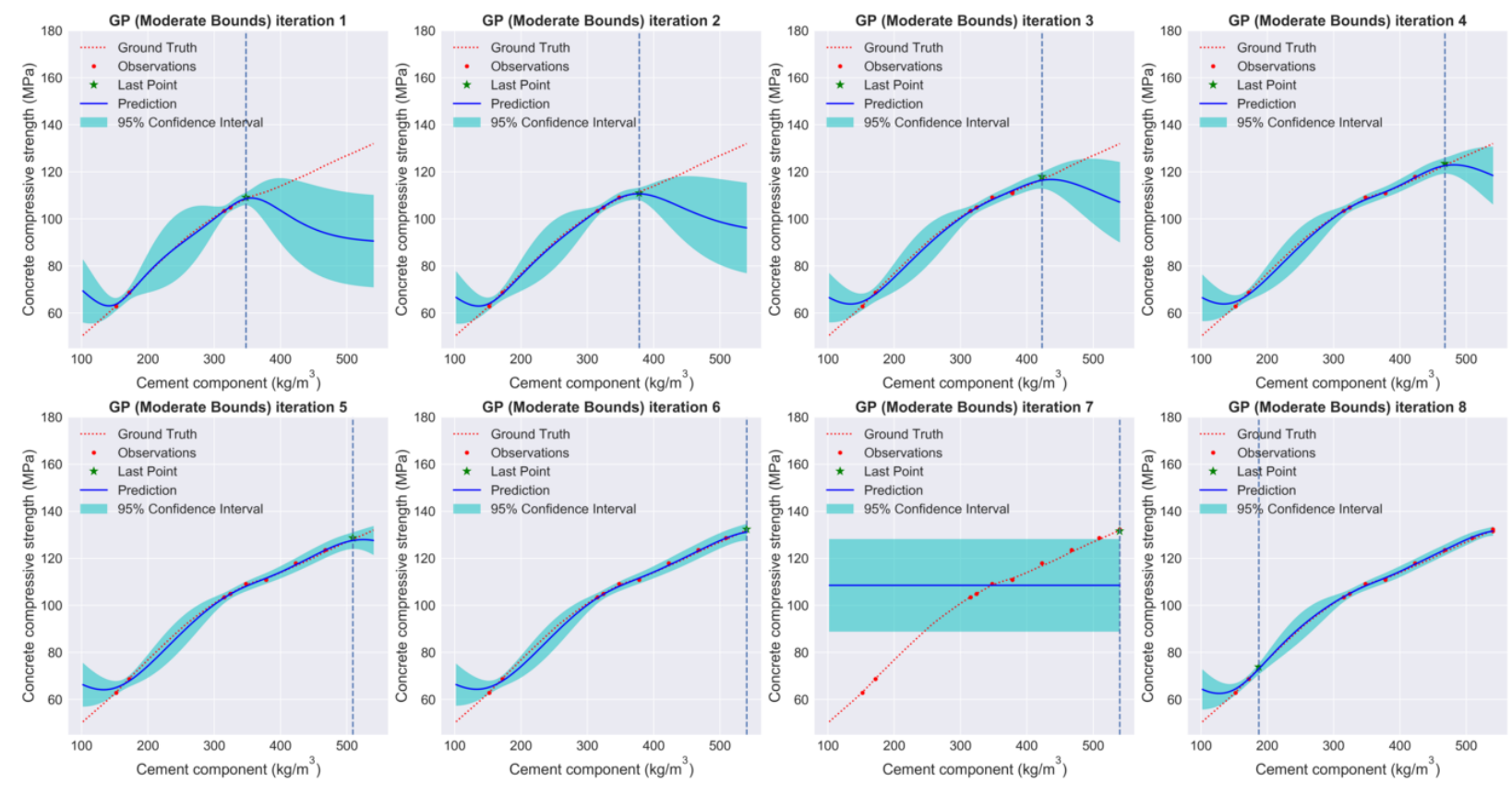

Figure. S5. Evolution of the prediction and uncertainty manifolds over the first few optimization iterations for the Gaussian Process surrogate model, with moderate bounds for the Matern kernel length scale. 

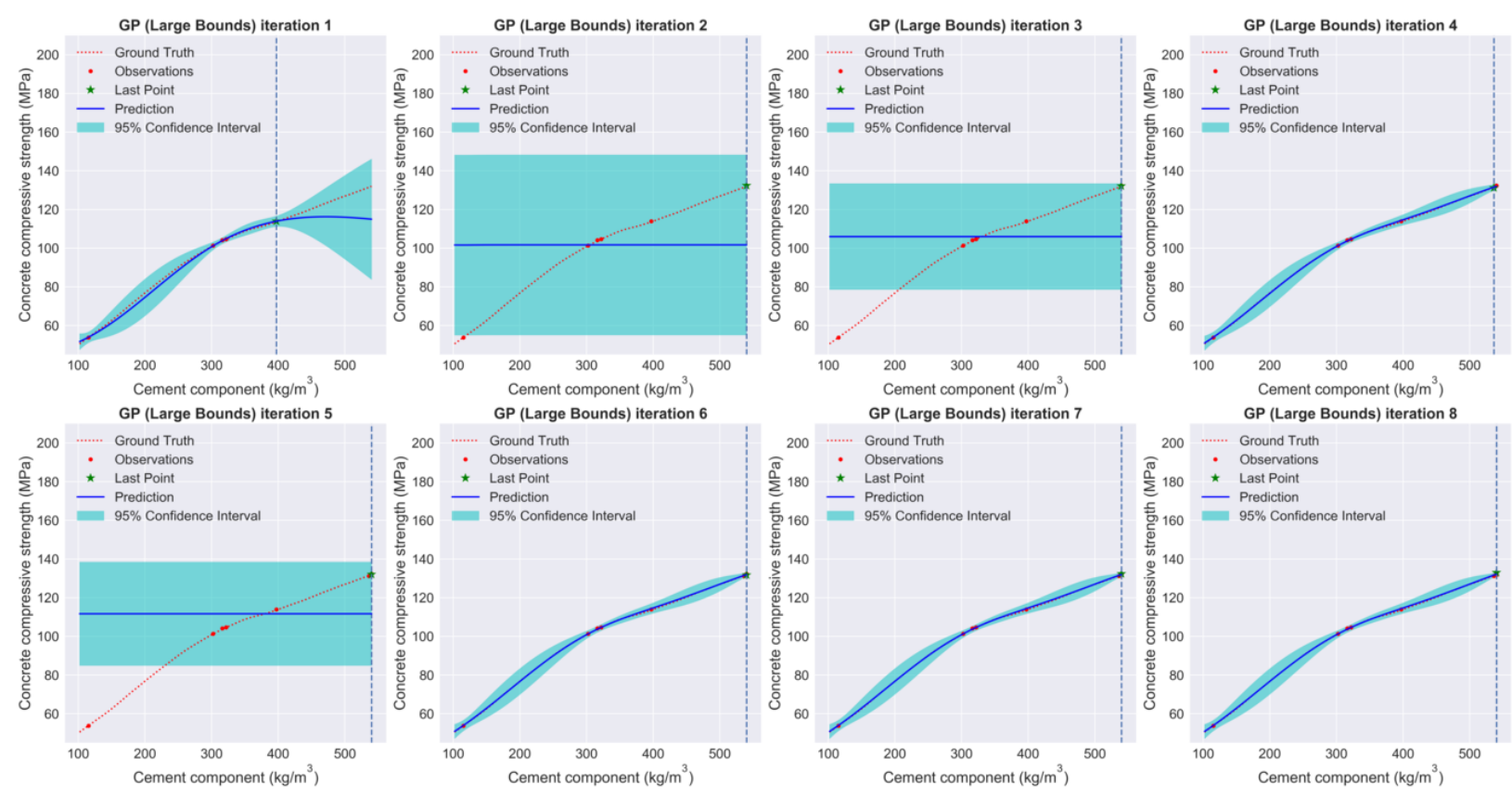

Figure. S6. Evolution of the prediction and uncertainty manifolds over the first few optimization iterations for the Gaussian Process surrogate model, with large bounds for the Matern kernel length scale.

\begin{tabular}{rrrrrr} 
& Ambient Temperature $\left({ }^{\circ} \mathbf{C}\right)$ & Exhaust Vacuum $(\mathbf{c m ~ H g})$ & Ambient Pressure (mbar) & Relative Humidity (\%) & Energy Output (MW) \\
\hline $\mathbf{0}$ & 14.96 & 41.76 & 1024.07 & 73.17 & 463.26 \\
$\mathbf{1}$ & 25.18 & 62.96 & 1020.04 & 59.08 & 444.37 \\
$\mathbf{2}$ & 5.11 & 39.40 & 1012.16 & 92.14 & 488.56 \\
$\mathbf{3}$ & 20.86 & 57.32 & 1010.24 & 76.64 & 446.48 \\
$\mathbf{4}$ & 10.82 & 37.50 & 1009.23 & 96.62 & 473.90
\end{tabular}

Figure. S7. Snapshot of the combined cycle power plant dataset, showing the inputs for operating conditions and the corresponding output target of the energy output. This is a simple dataset with just 4 input columns. 

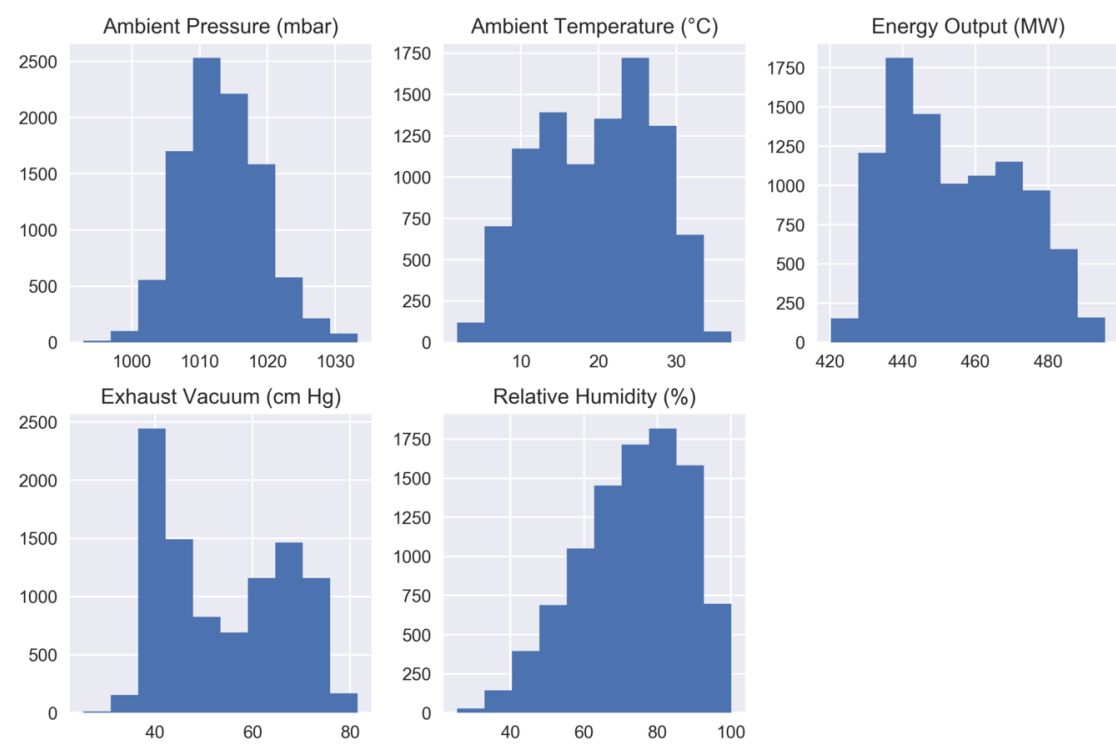

Figure. S8. Histograms illustrating the data distribution of the various inputs of the power plant dataset. In general, the data is well distributed, although the variations in the Ambient Pressure and Energy Output are small.

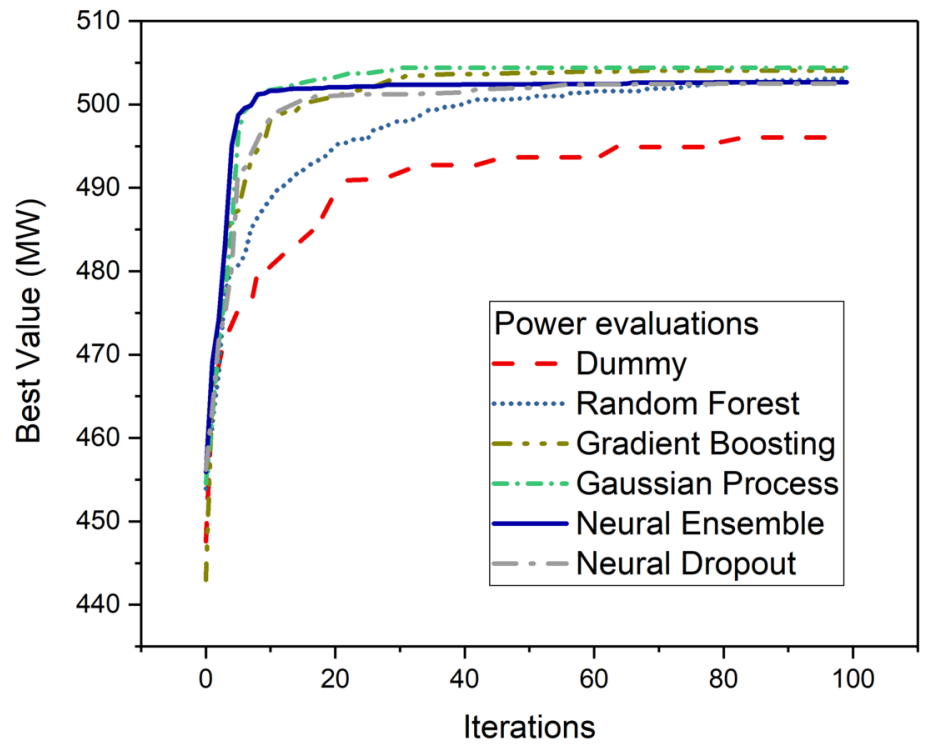

Figure. S9. Best power plant energy output evaluations averaged over $10 \mathrm{runs}$, using the trained Polynomial Regression (degree 2) algorithm as the predictive oracle and compared across different surrogate models. For this simple dataset, the Polynomial Regression turned out to be one of the better performing algorithms for extrapolative purposes. Here, there does not appear to be perceptible difference in the performance of the various models. 


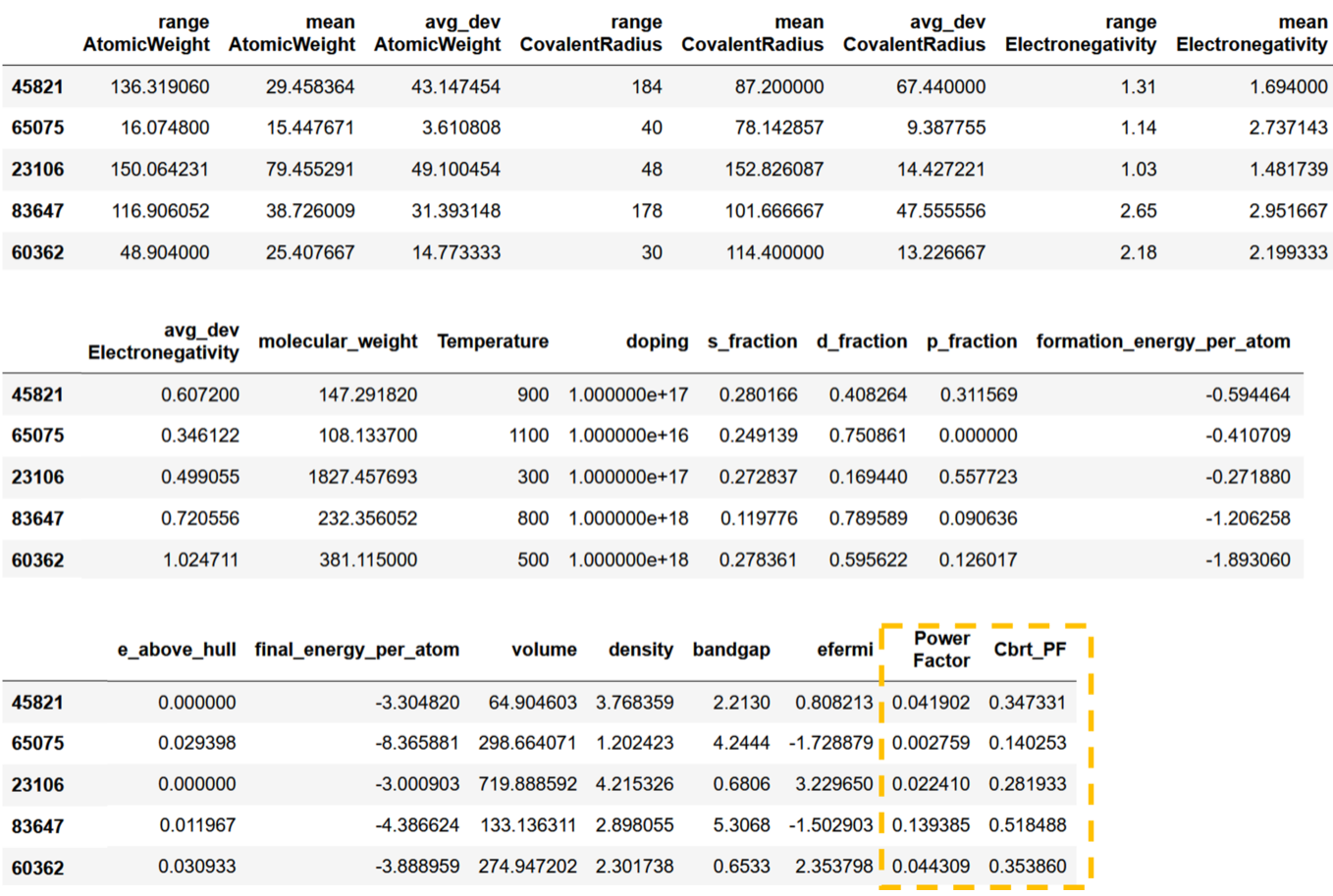

Figure. S10. Snapshot of the thermoelectrics power factor dataset, showing the inputs corresponding to various material electronic properties and the output target which is the cube root of the power factor. This is a significantly more complex dataset with 22 input columns. 

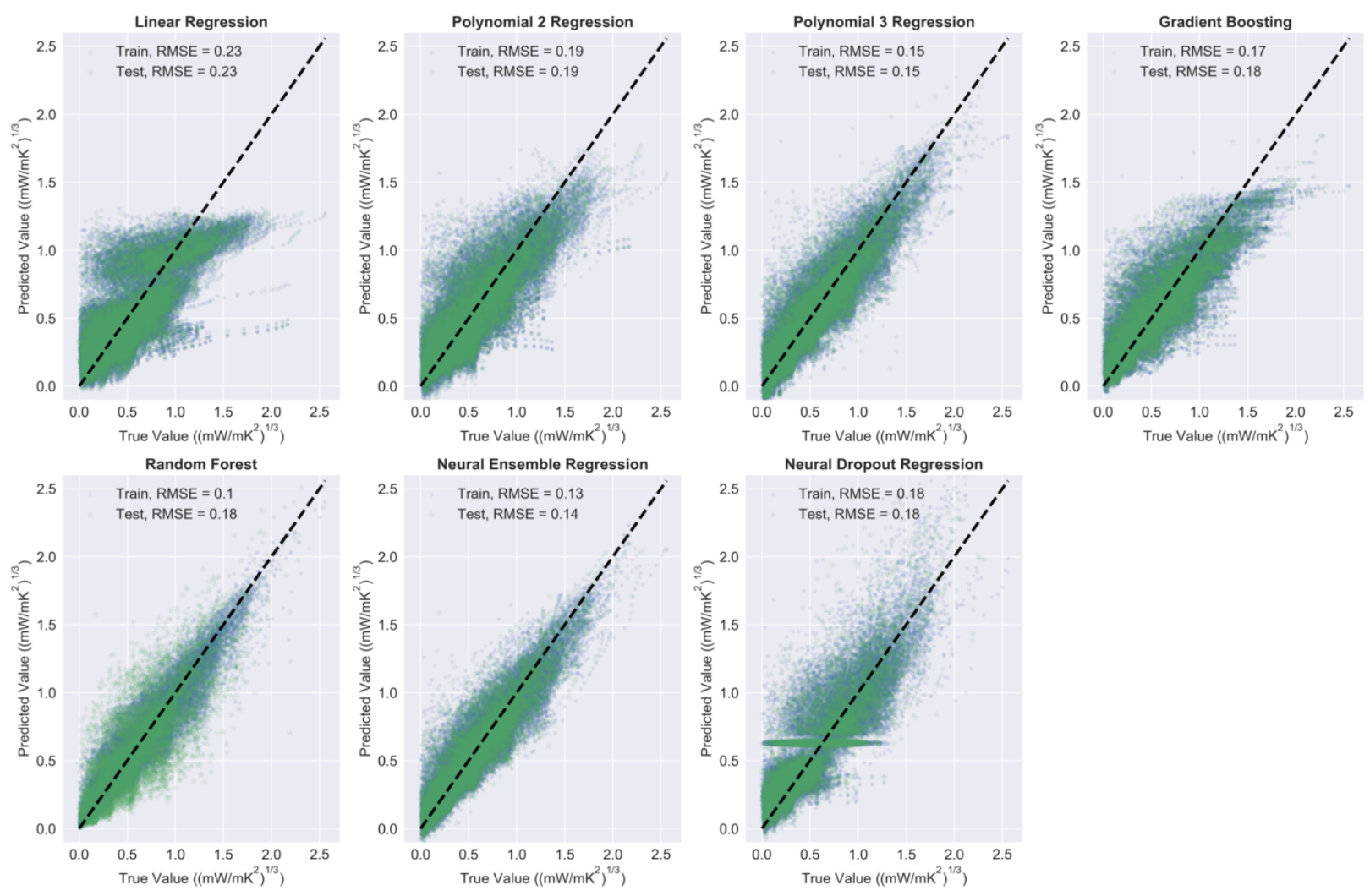

Figure. S11. Scatter plot visualizations of the actual thermoelectric power factor (cube root) vs values predicted by each of the trained regressors, with the training and test sets labeled separately. Also indicated are the RMSE scores for each regressor.
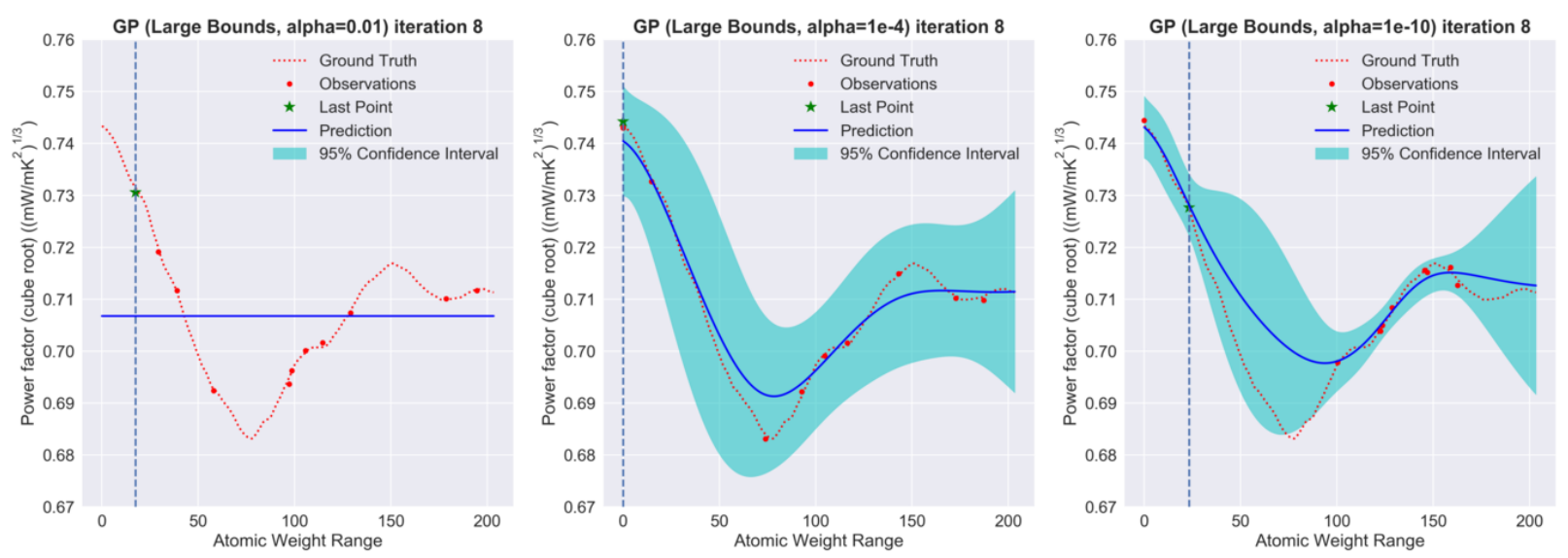

Figure. S12. Visualization of the prediction and uncertainty manifold for the Gaussian Process surrogate model applied to the thermoelectric dataset, for different values of alpha. The alpha parameter is an indication of the noise level inherent in the dataset. It should be noted that if alpha is set inappropriately, the 
GP is unable to accurately model the given data.

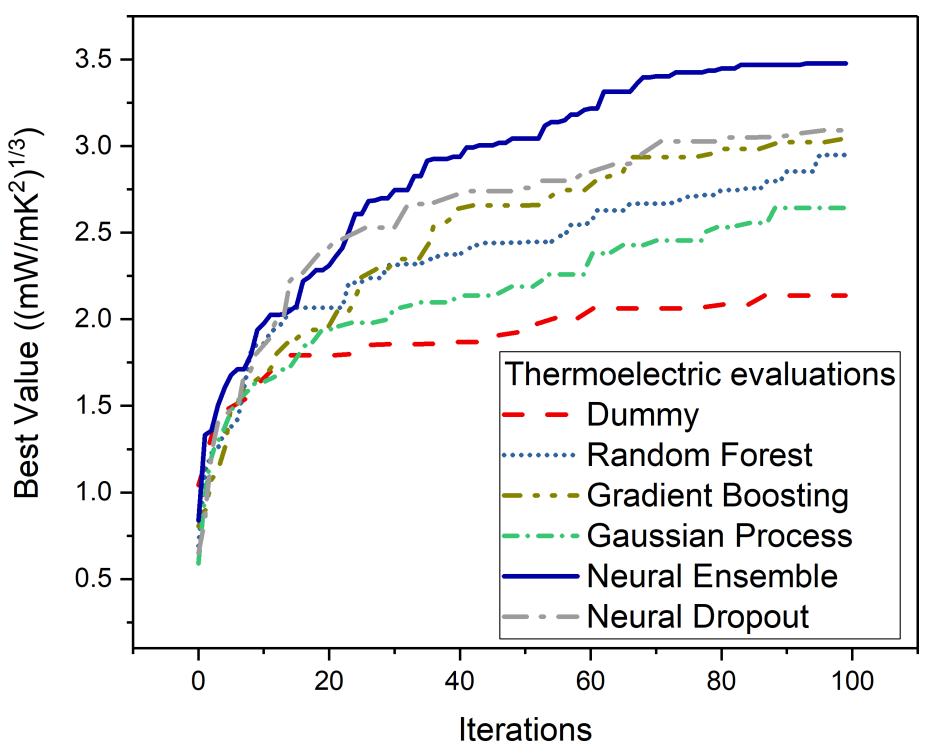

Figure. S13. Comparison of the speed of the optimization for the thermoelectric dataset with different surrogate models. Here, the Gaussian Process model parameters have not been optimized. With an alpha value of 2 (similar to that used for the concrete dataset), the GP model can barely outperform random search, whereas a tuned GP model can be the top performer. These results illustrate the importance of proper tuning of the GP parameters.

\begin{tabular}{lll}
\hline Index & Input Name & Lambda parameter \\
1 & Atomic Weight Range & 0.73 \\
2 & Mean Atomic Weight & 0.37 \\
3 & Atomic Weight Deviation & 0.50 \\
4 & Covalent Radius Range & 1.00 \\
5 & Mean Covalent Radius & 0.72 \\
6 & Covalent Radius Deviation & 0.88 \\
7 & Electronegativity Range & 1.14 \\
8 & Mean Electronegativity & 1.30 \\
9 & Electronegativity Deviation & 0.98 \\
10 & Molecular Weight & 0.01 \\
11 & Temperature & 1.00 \\
12 & Doping & -1.74 \\
13 & S Fraction & 0.33 \\
14 & D Fraction & 1.16 \\
15 & P Fraction & 0.72 \\
16 & Formation Energy Per Atom & 1.12
\end{tabular}




\begin{tabular}{lll}
17 & Energy Above Hull & -2.33 \\
18 & Final Energy Per Atom & 1.08 \\
19 & Volume & -0.70 \\
20 & Density & 0.47 \\
21 & Bandgap & 0.52 \\
22 & Fermi Level & 1.02 \\
\hline
\end{tabular}

Table S1. Fitted Yeo-Johnson Lambda parameters for the thermoelectric dataset inputs

\section{Figures with Data and Code}

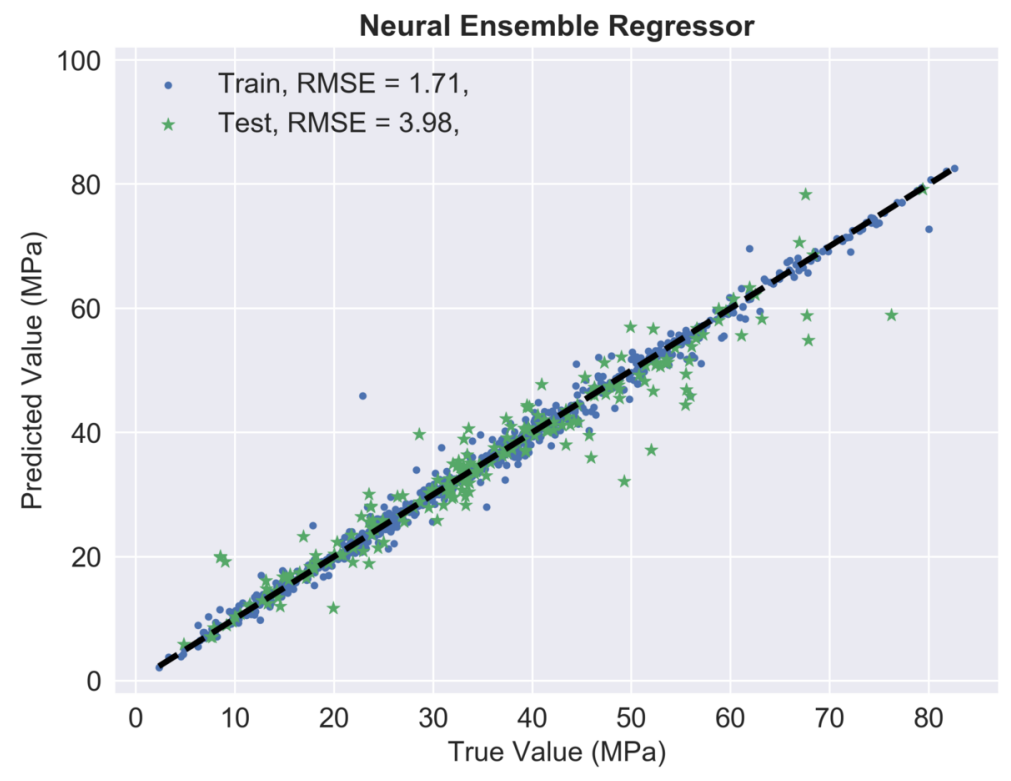

Figure 1: Concrete dataset machine learning compressive strength predictions using Neural Ensemble Regressor 


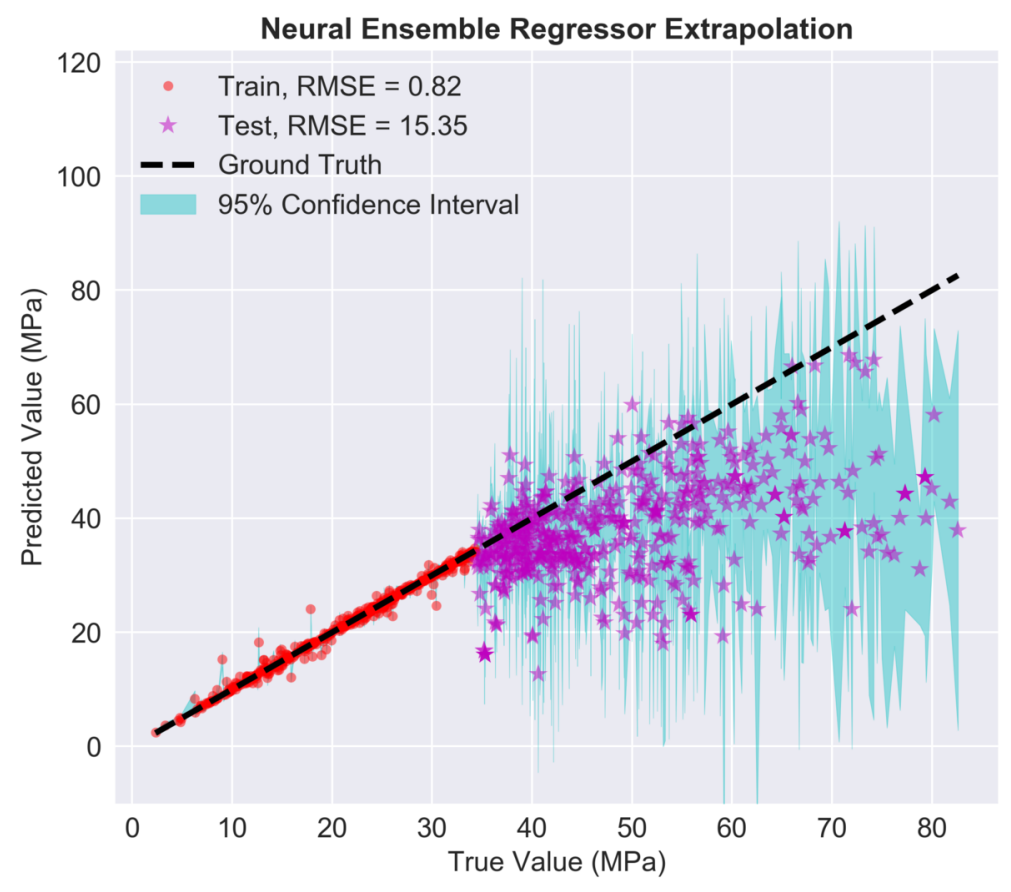

Figure 2: Visualizing the extrapolative performance of the Neural Ensemble Regressor 


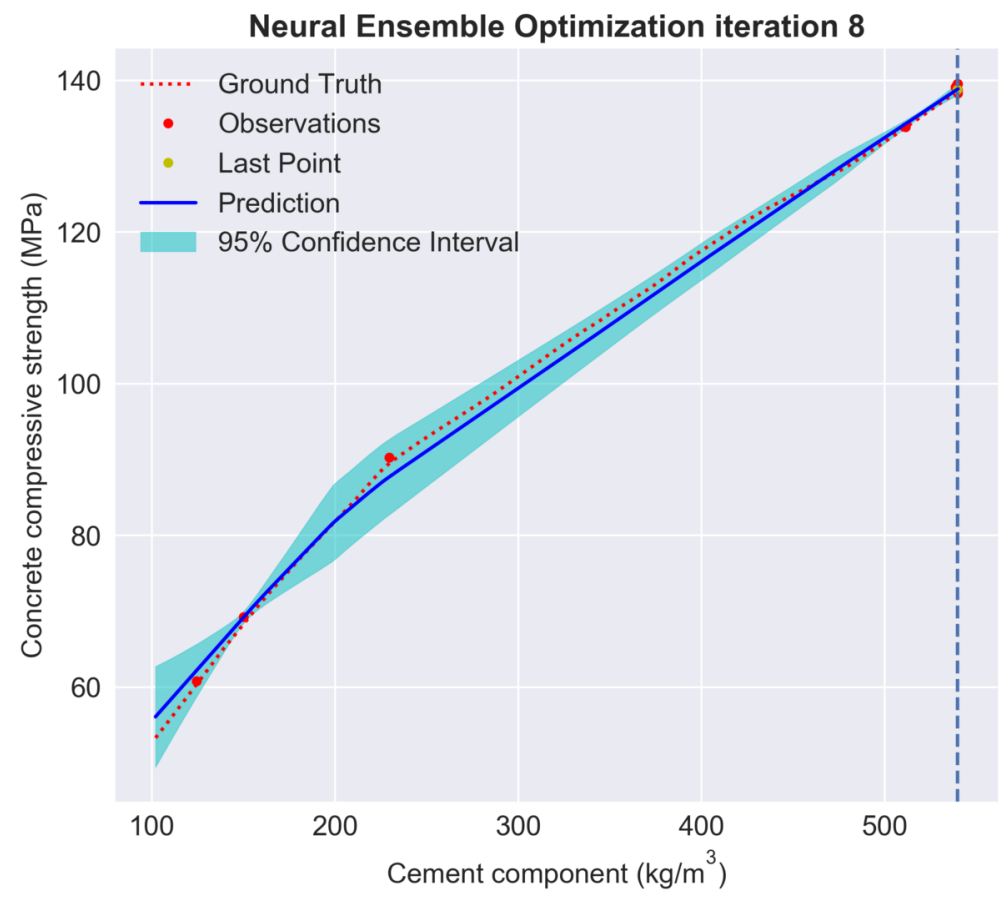

Figure 3: Visualizing the concrete Bayesian Optimization in 1D using the Neural Ensemble as the surrogate model

\section{Data}

Concrete compressive strength dataset

\section{Hosted file}

Concrete_Data.xls available at https://authorea.com/users/418278/articles/525086-supportinginformation-for-extrapolative-bayesian-optimization-with-gaussian-process-and-neuralnetwork-ensemble-surrogate-models

Thermoelectrics power factor dataset

\section{Hosted file}

cubic.xlsx available at https://authorea.com/users/418278/articles/525086-supportinginformation-for-extrapolative-bayesian-optimization-with-gaussian-process-and-neuralnetwork-ensemble-surrogate-models

Power plant output dataset

\section{Hosted file}

Power Plant.xlsx available at https://authorea.com/users/418278/articles/525086-supportinginformation-for-extrapolative-bayesian-optimization-with-gaussian-process-and-neural- 
network-ensemble-surrogate-models

\section{Code}

Concrete optimization (Jupyter ipython notebook)

\section{Hosted file}

Concrete Optimization.ipynb available at https://authorea.com/users/418278/articles/525086supporting-information-for-extrapolative-bayesian-optimization-with-gaussian-processand-neural-network-ensemble-surrogate-models

Thermoelectrics optimization (Jupyter ipython notebook)

\section{Hosted file}

Thermoelectric Optimization.ipynb available at https://authorea.com/users/418278/articles/ 525086-supporting-information-for-extrapolative-bayesian-optimization-with-gaussianprocess-and-neural-network-ensemble-surrogate-models

Power plant optimizaton (Jupyter ipython notebook)

\section{Hosted file}

Power Optimization.ipynb available at https://authorea.com/users/418278/articles/525086supporting-information-for-extrapolative-bayesian-optimization-with-gaussian-processand-neural-network-ensemble-surrogate-models

Synthetic functions optimization (Jupyter ipython notebook)

\section{Hosted file}

SynFn Optimization.ipynb available at https://authorea.com/users/418278/articles/525086supporting-information-for-extrapolative-bayesian-optimization-with-gaussian-processand-neural-network-ensemble-surrogate-models

Neural Ensemble regressor (python file)

\section{Hosted file}

NeuralEnsemble.py available at https://authorea.com/users/418278/articles/525086-supportinginformation-for-extrapolative-bayesian-optimization-with-gaussian-process-and-neuralnetwork-ensemble-surrogate-models

Neural Dropout regressor (python file)

\section{Hosted file}

NeuralEnsembleDropout.py available at https://authorea.com/users/418278/articles/525086supporting-information-for-extrapolative-bayesian-optimization-with-gaussian-processand-neural-network-ensemble-surrogate-models 